\title{
XPSSurfA: An Open Collaborative XPS Data Repository using the CMSShub Platform
}

\author{
Anders J. Barlow, Robert T. Jones, Andrew J. McDonald, Paul J. Pigram* \\ Centre for Materials and Surface Science and Department of Chemistry and Physics, School of Molecular \\ Sciences, La Trobe University, Melbourne, Victoria 3086, Australia
}

\begin{abstract}
$\underline{\text { Abstract }}$
X-ray photoelectron spectroscopy (XPS) is a widely used surface analysis technique employed in fundamental research, applied research, service laboratories and industry. Good quality analytical outcomes depend critically on spectral references. Many examples of XPS reference databases exist, including print editions, sets of spectral peak positions drawn from the literature, and digital archives and libraries. We report the development of a new digital XPS database comprising survey spectra and region spectra for a range of materials types, collected under a common set of analytical conditions. Each material is described using spectra collected at multiple pass energies with all photoelectron and X-ray induced Auger transitions represented. Detailed metadata are provided for each material and each spectrum, presented using a schema that incorporates the ISO 16243 and 14976 standards and extensions developed in this work. Spectra are shared under a Creative Commons International (4.0) attribution, non-commercial licence (CC BY-NC) in Kratos (.dset), VAMAS (.vms) and XML (.xml) formats. It is intended that reference spectra be imported directly into XPS data analysis software packages for reference and comparison purposes, matching either the peak or transition of interest and the instrument pass energy. The database is flexible and scalable in structure and has the potential to become a core XPS reference resource.
\end{abstract}

Keywords: XPS, database, eResearch, metadata, HUBzero

This is the author manuscript accepted for publication and has undergone full peer review but has not been through the copyediting, typesetting, pagination and proofreading process, which may lead to differences between this version and the Version of Record. Please cite this article as doi: $10.1002 /$ sia. 6417

This article is protected by copyright. All rights reserved. 
*Corresponding author email: P.Pigram @ latrobe.edu.au

This article is protected by copyright. All rights reserved. 


\section{Introduction}

X-ray photoelectron spectroscopy (XPS) is an essential surface analytical technique supporting a broad range of research areas. We estimate that there are several thousand XPS instruments operating in academic and industrial research laboratories around the world. The well-established market position of XPS instrumentation and recent rapid uptake in sectors focussed on polymeric materials ensures that there is a high level of exposure of new analysts from many disciplines to the technique. Laboratory analytical spectroscopies such as vibrational spectroscopy (UV/Visible/NIR), mass spectrometry, and chromatography (GCMS/LCMS) are well served with openly available spectral databases allowing users of an instrument to compare a given unknown with a known spectrum. For example, the National Institute of Standards and Technology (NIST) maintains the NIST Chemistry WebBook, which is reported to contain over 80,000 reference spectra from common compounds and a number of laboratory techniques ${ }^{1}$, but not for XPS. By comparison, fewer such resources are available to the surface analysis community.

Reference sources for the XPS analyst generally take the form of numerical databases. NIST hosts an online XPS database that compiles data from peer-reviewed scientific publications from as early as 1968, with version 1.0 originally collated by Dr Charles Wagner ${ }^{2}$ and now version 4.1 by Dr Cedric Powell and colleagues ${ }^{3}$. The NIST XPS database categorises numerical peak-position data by element and transition and the chemical state or molecular formula of the specimen under study in the cited publication. All data are critically evaluated by the curators, ensuring that only data from adequately described spectra and materials are included. It is a numerical database, meaning that only the peak positions are provided. No spectral data are stored for a user to view or access. A citation for the original research is included, so that the reader can inspect the original spectra if desired. A similar online XPS resource is LaSurface ${ }^{4}$, which tabulates XPS binding energies for elements and compounds in a searchable database similar in structure to the NIST database. Thermo Scientific host the website XPS Simplified, which includes a version of their Knowledge Base ${ }^{5}$. The online version is a numerical database comparable to the aforementioned sources, but one that provides greater practical details about the collection and interpretation of data from a chosen 
element/material, guiding the analyst through the acquisition of XPS data. References are provided when information is presented from external sources, but the spectra shown are collected by application scientists from Thermo Scientific using company instrumentation. A powerful feature of this database is the ability to use it within the Thermo Scientific Avantage Data System. Users can import data from the Knowledge Base directly into their experimental frame, allowing immediate comparison with XPS data as it is being acquired on the instrument.

Many texts are available that include numerical XPS databases and, in some cases, spectra. A common and popular reference is the Handbook of X-ray Photoelectron Spectroscopy published by Physical Electronics (PHI) ${ }^{6}$. The PHI handbook provides wide-energy survey spectra for all the elements from lithium to uranium using $\mathrm{Al} \mathrm{K \alpha}$ and $\mathrm{Mg} \mathrm{K \alpha}$ excitation, and includes high-resolution spectra of the most intense peaks and some Auger features. Also included are numerical databases of chemical states and Auger parameters, along with their respective references, much of which came from Wagner and is included in the NIST Database. The Scienta ESCA300 polymer database is focused on XPS spectra from organic polymers and was acquired and collated by Graham Beamson and David Briggs ${ }^{7}$. This database contains spectra from 111 polymers, collected under high resolution and high signal conditions from a single instrument. Given its focus on polymeric materials, the text comprises not only wide and narrow scan spectra from multiple regions on each polymer, but also some chemical structure information, guidance on peak fitting, and figures of merit regarding expected X-ray damage. Since the book went out of print, the original datafiles that made up the spectra in the text have been made available in a number of electronic formats, including the ISO 14976 VAMAS standard ${ }^{8}$.

Along with the Beamson and Briggs database, there are other electronic collections of spectra available. XPS International offers a number of packages that include a significant amount of reference spectra with a dedicated data analysis software package ${ }^{9}$. The American Vacuum Society (AVS) publishes Surface Science Spectra, a journal dedicated to disseminating peerreviewed surface analytical data including XPS, UPS and AES spectra. Articles are well described with metadata pertaining to sample preparation methods and instrument details and 
conditions. Recently it was announced that this collection of data would be made available through a new online tool, whereby researchers can browse the collection of published spectra ${ }^{10}$. The Surface Analysis Society of Japan provides COMPRO, the Common Data Processing System for XPS and AES spectral analysis ${ }^{11}$. This software package incorporates two databases totalling more than 3,300 XPS and AES spectra.

The Centre for Materials and Surface Science (CMSS) at La Trobe University hosts a suite of surface analysis instrumentation including two Kratos Analytical XPS instruments. These instruments provide an analytical service for academia and industry, and support a variety of university-led research projects.

CMSS has developed a collaboration environment known as CMSShub ${ }^{12}$, based on the HUBzero platform originated by Purdue University ${ }^{13}$. CMSShub incorporates facilities for group and project-based collaboration, communications and information sharing. The XPS database we describe in this article is hosted via CMSShub and takes the form of a HUBzero application.

Motivation existed within CMSS to build a repository of XPS data for materials that were often analysed in our XPS instruments, or that were often used in comparisons against novel materials, or materials deposited/fabricated using novel methods. It appeared prudent to collect data once, and apply those data across multiple analyses and projects. Thus, there was impetus to create a repository of XPS data with desirable characteristics:

- All data collected entirely on instruments within CMSS

- Identical conditions used for all analyses set forth by a prescribed analysis protocol

- Data stored in formats that enable rapid and easy dissemination

- All metadata associated with each analysis is stored along with the data

- Data repository stored in the cloud, with redundancy, and accessible from anywhere.

In addition, the database has been developed with an open access structure in mind to facilitate collaboration and sharing of these data. The database is consistent with the guiding principles of FAIR Data; that is, that data be Findable, Accessible, Interoperable and Reusable $^{14}$. 
The result is XPSSurfA ("XPS Surfer", with surfer stylised to reflect the Surface Analytical context of the database; cmsshub.latrobe.edu.au/xpsdatabase), a database of XPS spectra collected entirely on the two XPS instruments within CMSS at La Trobe University, and made freely available to researchers through a Creative Commons (CC) open access license. XPSSurfA is an application developed within the HUBzero environment and accessed through CMSShub. Researchers can create an account on CMSShub, which in turn provides access to XPSSurfA, as well as the powerful collaborative nature of the HUBzero landscape. XPSSurfA is searchable via keyword terms, experimental details, such as source energy or instrument type, and material identifiers, such as chemical abstracts service (CAS) ID. Every record has a unique identifying number associated with it and there is a facility to create unique digital object identifiers (DOIs) for every record in XPSSurfA. The entire database is linked to a single DOI and a record on the Research Data Australia (RDA) repository. All data are conveyed to users via the ISO standard VAMAS format (ISO 14976:1998 ${ }^{15}$ ), a constructed eXtensible Mark-up Language (XML) format, and the original proprietary binary format direct from the XPS instrument. Every record and file on the database is described by rich metadata collected from the material properties and provenance, the sample handling and preparation procedures, the analysis protocols, and the instrumental conditions at the time of data collection. Researchers can find records on XPSSurfA, view the records in their browser window, view all metadata associated with a given record, decide if the data is valuable to their research, and download the data to be compared to, and ultimately published alongside, their own research data, with appropriate attribution set forth by the CC license.

Herein we describe XPSSurfA in detail, and our underlying CMSShub, HUBzero and Remote Laboratory Instrumentation (RLI) systems. We describe in full the data acquisition and sample preparation protocols, the metadata schema, the chosen data transfer formats, choice of open access license, and usage statistics for the database thus far. 


\section{Architecture of the Database}

\subsection{CMSShub and ANFFhub}

CMSS operates and manages two online collaborative resources: CMSShub and ANFFhub, which are implemented to enhance connectivity between the surface scientists within CMSS, who operate and manage the instrumentation, and the researchers and collaborators that request analyses. Both CMSShub and ANFFhub are instances of HUBzero, which is a comprehensive online environment for scientific collaboration developed by Purdue University ${ }^{13}$. The HUBzero environment enables the creation of online resources, training tools, collaboration groups, forums, news feeds, and many other features, with users of the system able to be assigned to certain groups based on privilege levels or membership. For example, in our instance CMSShub, users can be assigned to a research group set up exclusively for their institution, and only those group members can see the content within that group. Additionally, users across all institutional groups can be assigned to an instrument user group, allowing them access to the content for a given instrument or technique. In this way, we can maximise our interactivity with collaborators and keep all users up to date with news from the facility, the latest techniques, and improvements in software or processing through a single online system.

The HUBzero web environment is designed with a LAMP architecture:

- Linux OS - Debian GNU/Linux 7 (wheezy)

- Apache 2 web server - sends HTML pages to remote clients

- MySQL database - store all web server content

- PHP scripting - to interact with the MySQL database and generate HTML pages

HUBzero is written with a fully object-oriented application programming interface (API). This is a very large library of PHP and JavaScript routines, based on Joomla, which can be called by PHP scripts to generate web content and to setup and access MySQL databases. The CMSShub and ANFFhub instances of HUBzero run on virtual machines (VMs) allocated to the NeCTAR Cloud, a resource provided for Australian researchers by the National eResearch Collaboration Tools and Resources project (Nectar) ${ }^{16}$. 


\subsection{XPSSurfA Application on CMSShub}

XPSSurfA is a custom application written for integration in the HUBzero collaboration environment utilising the HUBzero API. It is implemented as a 'Component', which is a complex extension to the HUBzero environment acting as a separate application. It has its own functionality, MySQL databases and presentation through CSS files. The XPSSurfA application has been structured in the Model-View-Controller (MVC) software design pattern. The Models establish the data objects and provide functionality for accessing, storing and manipulating the data objects in the MySQL database. The data objects in this case are the XPS datasets. The Controllers respond to user actions via the web, such as page requests. The controller interprets the request, determines the appropriate response and triggers the Model to manipulate the data accordingly, passing the data to the View. The View actually displays the data to the client, contains the entire HTML formatting and interacts with the CSS style files for the web site. For example, cmsshub.latrobe.edu.au/xpsdatabase/xpsrecords/view/135 triggers the Controller "xpsrecords" to access the Model for \#135, obtain the available spectra from the database, sort the spectra, and then pass to the View "xpsrecords". Upon clicking 'View Spectra' a different Controller is initiated, which accesses all the spectra from the Model, generates interactive plots using JavaScript, gathers metadata from the Model database, etc., and sends it all to the View "view_many". Interactions with the spectral analysis tool are performed via JavaScript uploaded to, and running on, the client-side browser.

\subsection{Remote Laboratory Instrumentation (RLI)}

CMSS operates and manages an in-house developed system called Remote Laboratory Instrumentation (RLI), a web server that allows users to request and book jobs on the instruments within CMSS, and manage, store and access the generated data. RLI exists across a number of Linux servers running CentOS 6.9, also using a LAMP architecture. Like CMSShub, it runs on a number of virtual machines (VMs) allocated to the NeCTAR Cloud resource. 
RLI manages all user account information, analysis requests, instrument scheduling, data uploading and access, and user billing aspects of CMSS. It is purpose-built to suit the needs of the facility. Once a user account is created, the user may request surface analysis on any of the CMSS instruments. This request takes the shape of an online form that, when submitted, writes the details into the MySQL database. An example Surface Analysis Request form is given in Figure S1 in the supplementary information. The request contains information about the user, the technique requested, and details about the sample(s) and the desired analysis. CMSS staff then approve the request (if satisfactory), and assign billing details and the most appropriate CMSS instrument for the work. The instrument can then be booked to perform the work, at which point the operator assigns the request to the booking. All details are then carried across to the Surface Analysis Record (logsheet). This form is for the instrument operator to input experimental details at the time when the data are collected for the request, an example of which is given in Figure S2. These two forms underpin a significant portion of the metadata collection for XPSSurfA. Once work is completed, the data are uploaded from the instrument PC to the RLI storage gateway on the cloud, where the instrument operator and the original analysis requestor can access the data files through the RLI Storage Gateway. No other users can access these data, only those who were initially associated with the Surface Analysis Request and Record.

\section{$2.4 \quad$ RLI, CMSShub and XPSSurfA}

XPS datasets collected for the purposes of inclusion in XPSSurfA are uploaded from the RLI web server to CMSShub for listing in the XPSSurfA application. The metadata that appears in XPSSurfA are automatically extracted from the RLI Surface Analysis Request and Record forms and the VAMAS files generated by the Kratos instruments. The VAMAS files, which also contain the spectra datasets, are translated into XML files using a custom written parser. Custom PHP scripts then gather the metadata from the RLI MySQL databases and from the parsed VAMAS files, process it appropriately, and then send it via SSH (secure shell) protocol to the CMSShub MySQL databases. The spectra are transferred and stored as XML files on the CMSShub. The parser is based on the VAMASXML code originally written by Conal Touhy ${ }^{17}$. 


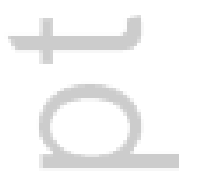

4 
3. Creation of the Database

\subsection{Instrumentation and Calibration}

CMSS operates two commercial XPS instruments supplied by Kratos Analytical (Manchester, UK), the AXIS Nova and AXIS Ultra. Each uses a monochromatic Al Ka X-ray source with a nominal excitation energy of $1486.69 \mathrm{eV}$, and a maximum output power of $300 \mathrm{~W}$ (when operated at $15 \mathrm{kV}, 20 \mathrm{~mA}$ emission). The source, monochromator and sample are positioned on a $500 \mathrm{~mm}$ Rowland circle. The analyser for spectroscopy is a hemispherical analyser (HSA) design with a $165 \mathrm{~mm}$ mean radius, while parallel XPS imaging is achieved by passing the electrons into a spherical mirror analyser (SMA) positioned on top of the HSA outer hemisphere. The source-analyser angle is 54.6 (Nova) and 60 degrees (Ultra). Electron detection is performed by a 2-dimensional delay-line detector (DLD) comprising a multichannel plate (MCP) stack above orthogonal delay-line anodes, enabling high count-rate scanned spectroscopy, snapshot spectroscopy, and 2D parallel imaging. The detector assembly is positioned at the exit slit of the HSA. The MCP voltage is set $50 \mathrm{~V}$ above the 'knee' in a plot of counts-per-second (CPS) vs detector voltage. Insulating or otherwise nonconductive materials can be analysed in these systems by use of a low-energy electron source. This charge neutralisation system is positioned coaxially with the electrostatic lens column above the sample surface. The sample is positioned within the field of a magnetic immersion lens that serves to focus emitted photoelectrons into the electrostatic lens column, giving rise to greatly increased signal, and assisting in the charge neutralisation of the surface through the spiral motion of the low energy electrons in the magnetic field. Each instrument is equipped with a monoatomic argon ion source, the Minibeam III on the AXIS Ultra and Minibeam IV on the AXIS Nova, for the sputter cleaning of inorganic and metallic materials before analysis. The Nova analysis chamber achieves a base pressure of better than $5 \times 10^{-10}$ mbar, while the Ultra reaches $1 \times 10^{-10}$ mbar. In our laboratory, the Nova is used for high-throughput routine surface analysis, as well as depth-profiling, angle-resolved and imaging analyses. The Ultra on the other hand, with its attached radial distribution chamber, is used for more complex experiments and research, such as those requiring heating and cooling in the entry and analytical positions, gas dosing, crystal cleaving, and the insertion of 
samples into the system under inert environments (such as argon and nitrogen) within an acrylic glovebox.

The instruments are calibrated using the ISO standard for energy scale calibration in surface chemical analysis (ISO 15472:2010 ${ }^{18}$ ). Samples of high-purity gold, silver and copper foils are ion sputter cleaned to remove contamination and oxide layers, and high resolution $\mathrm{Au} 4 \mathrm{f}$, $\mathrm{Ag} 3 \mathrm{~d}$ and $\mathrm{Cu} 2 \mathrm{p}$ spectra are collected for a range of analyser modes to correct the span and offset of the measured energy scale. The monochromator and X-ray source are tuned to give the narrowest profile for the $\mathrm{Ag} 3 \mathrm{~d}_{5 / 2}$ peak with specification being $\leq 0.50 \mathrm{eV}$. Table 1 gives the energy scale calibration and Table 2 gives the energy resolution optimisation of both of these spectrometers at the time of the generation of the database.

\subsection{Material preparation and mounting}

In the context of sample preparation, materials analysed for the database may be broadly categorised as metals, solid non-metal materials (metalloids, semi-metals and semiconductors), polymers and powders/particulates. In addition, a set of adhesives commonly used in surface analysis have been analysed. Each category was treated differently to maximise the likelihood of excellent data acquisition once introduced into the vacuum system. The ISO 18116:2005 standard ${ }^{19}$ was followed as the basis for all sample-handling protocols.

Metal foils afforded the simplest preparation ex situ (note that here ex situ refers to the ISO 16243:2011 ${ }^{20}$ standard definition of outside the analytical system). Samples of the highest-purity that is reasonably purchased were cut to a size of approximately $10 \mathrm{~mm} \times$ $10 \mathrm{~mm}$, then cleaned in an ultrasonic bath using either isopropanol or ethanol to remove gross contamination. These foils were then mounted into a specially designed and manufactured back-mount holder (pictured in Figure 1) that enables a simple and robust mechanical and electrical connection to the surface, and mounts all sample surfaces to the same height, facilitating easy z-height alignment once inside the system. 


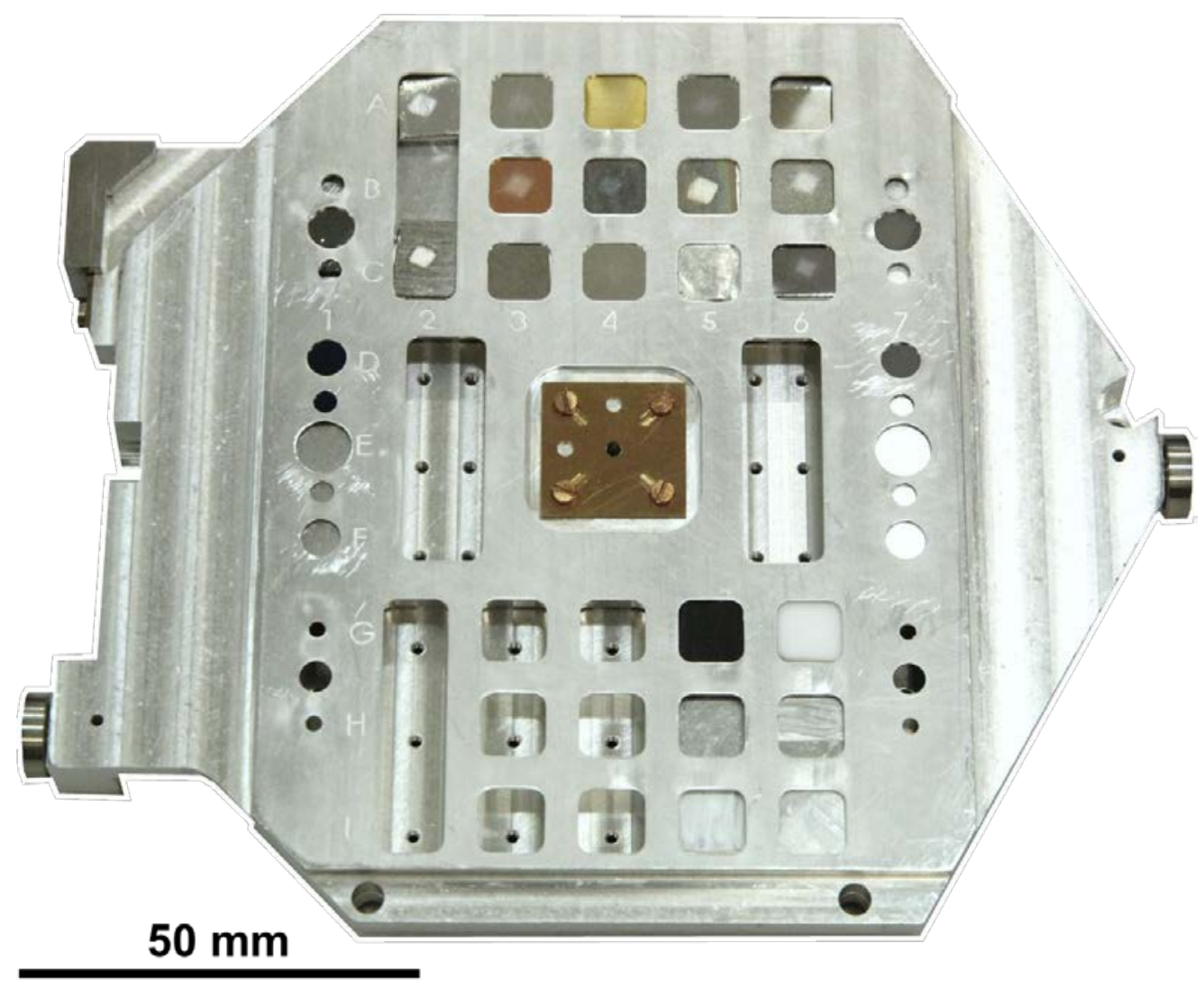

Figure 1 - Custom back-mount sample platen for the AXIS Nova system designed and manufactured in-house.

Materials categorised as solid non-metals included highly oriented pyrolytic graphite (HOPG), diamond, boron-doped diamond (BDD) and silicon. Each had a distinct preparation method before mounting in the back-mount holder. The HOPG sample $(10 \mathrm{~mm} \times 10 \mathrm{~mm})$ was freshly cleaved using adhesive tape a number of times to expose a pristine graphite layer. No further preparation was performed prior to mounting. BDD was mounted as received. The diamond sample $(5 \mathrm{~mm} \times 5 \mathrm{~mm})$ had been cleaned using a hydrogen plasma treatment before being received at CMSS. The sample was mounted into the AXIS Ultra using a molybdenum sample stub and a tantalum mask incorporating a $3 \mathrm{~mm}$ aperture through which the sample surface was accessible. Silicon samples (including silicon with native oxide and a wafer with $100 \mathrm{~nm}$ silicon dioxide layer) were treated in a similar way to metals, with solvent sonication to remove contamination before mounting. 
The choice of preparation method for polymers depended on the form of the polymer used. For polymers in the form of sheets/films, the surface was scraped thoroughly using a new scalpel blade, with each blade only used for a single sample. Sections of $10 \mathrm{~mm} \times 10 \mathrm{~mm}$ were then cut with scissors cleaned in isopropanol and back-mounted for analysis. Many polymers in the database were received in the form of small pellets, often highly variable in shape. To ensure a pristine and flat surface for analysis, the pellets were held in tweezers and cleaved using a new scalpel blade on opposing faces to ensure a flat base for mounting and for analysis. These pellets were then placed into specially designed powder well plates that screwed directly to the AXIS Nova platen.

Powder and particulate samples sometimes necessitated the use of an adhesive for mounting to minimise the risk of cross contamination of sample mounting and the instrument, though whenever possible adhesives were avoided. In most instances, powders were simply pressed into a powder well plate (Fig. 2) using a cleaned stainless steel spatula and transferred into the instrument for analysis.

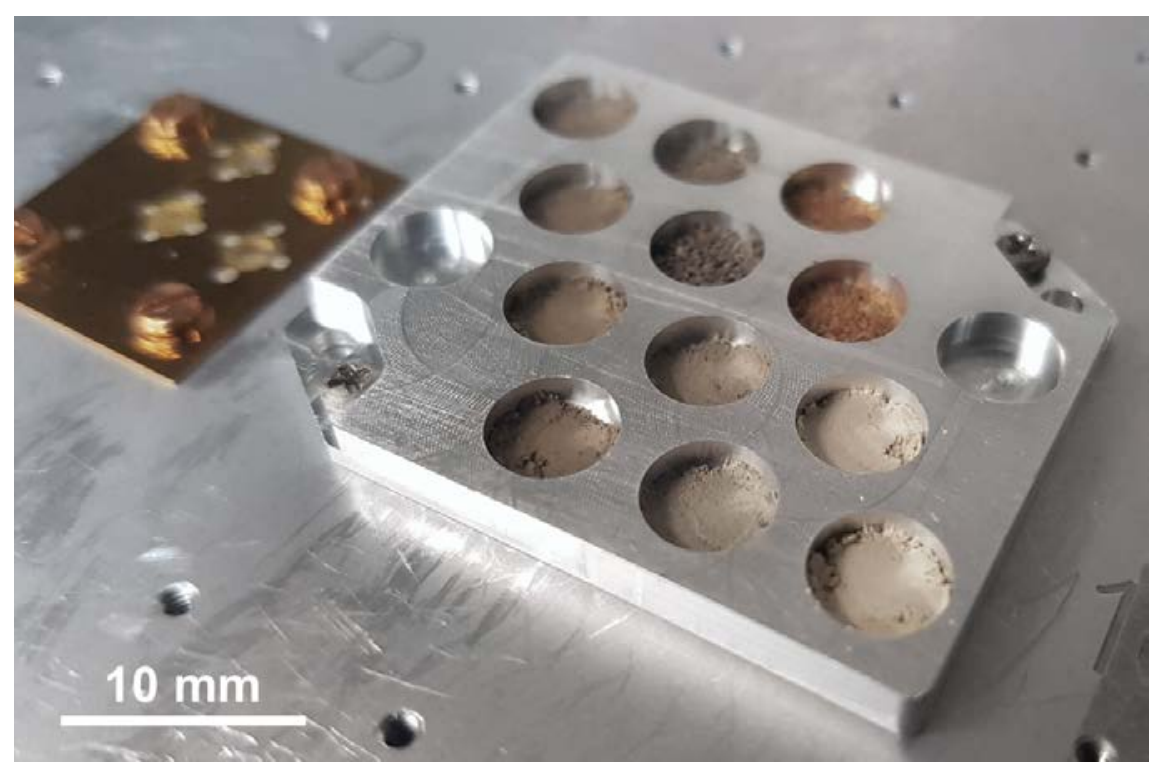

Figure 2 - Powder well plate mounted to the AXIS Nova 'plain' platen. Powder and particulate samples could be pressed into the wells, minimising cross-contamination of samples. 
Commonly used adhesives in surface analysis were also added to the database including carbon tape (from two sources), carbon tabs (often used in SEM analysis), 3M Scotch Tape type 665 (known to contain low amounts of silicone, sometimes used in ToF-SIMS analysis for this reason), and conductive graphite and silver paints. The tapes were cut to size and mounted for analysis, while the paints were spread onto the mounting surface.

\subsection{Data Acquisition}

A protocol for data acquisition was developed at the inception of the database and is described in detail in Table 3. By defining clearly from the outset key acquisition parameters and applying them throughout every analysis, we achieve a database that is cohesive and selfconsistent.

Every element and transition region in the accessible binding energy range is collected, including Auger transitions and the valence band. For materials that do not display sensitivity towards X-ray radiation (for example, metals) an X-ray source power of $225 \mathrm{~W}$ is used to increase total signal, but for most materials this is reduced to $150 \mathrm{~W}$. For typical analyses within CMSS, the source is operated at this lower power. The analyser is operated in fixed analyser transmission (FAT) mode, with an analytical area of $300 \times 700 \mu^{2}$, determined by the Field of View 1 (FoV1) lens mode with the Slot aperture on the AXIS Nova, and Hybrid lens mode (Slot) on the AXIS Ultra. Data are collected at $90^{\circ}$ take-off angle relative to the surface plane (take-off angle is defined by ISO 18115:2001 ${ }^{21}$ ).

Two survey spectra are collected for every dataset, a 'standard' survey collected at pass energy (PE) $160 \mathrm{eV}$ with a $1 \mathrm{eV}$ step size, and a so-called 'St Malo' survey ${ }^{22}$ collected at PE $80 \mathrm{eV}$ and step size $0.4 \mathrm{eV}$. This latter survey type is named after the $34^{\text {th }}$ IUVSTA Workshop ${ }^{23}$, held in St Malo, France, during which Technical Group C recommended the use of a survey with $0.4 \mathrm{eV}$ step size up to $1350 \mathrm{eV}$ binding energy for Al Ka radiation. A St Malo survey is often used in our laboratory when collaborators do not specifically request high-resolution spectra, and simply want quantification from a survey spectrum, as it can also be used for peak-fitting clear-cut chemical shifts. The benefit of the reduced step size and increased resolution from the lower pass energy is evident in Figure 3 for the $\mathrm{C} 1$ s region of 
poly(ethylene terephthalate) (PET). Three spectra are presented from the $160 \mathrm{eV}$ survey, the $80 \mathrm{eV}$ St Malo survey, and the $20 \mathrm{eV}$ high resolution scan. Peak fitting the latter two spectra clearly gives excellent agreement, and is also in good agreement with values given in the Beamson and Briggs database for $\mathrm{PET}^{7}$ and the expected 3:1:1 ratio of the components.

High-resolution spectra are collected from every element and transition region accessible for multiple pass energies: $20 \mathrm{eV}, 10 \mathrm{eV}$ and $5 \mathrm{eV} \mathrm{eV}$ (and additional spectra, such as at $40 \mathrm{eV}$ $\mathrm{PE}$ may be added in future analyses). The step size is set to $0.1 \mathrm{eV}$ for these spectra. X-ray induced Auger transitions are collected only at $20 \mathrm{eV}$ PE as these regions tend to be broad and lower intensity, thus no gain is made by using lower pass energies. Similarly, the valence band region is only collected at $20 \mathrm{eV}$ PE. The step size is typically set to $0.2 \mathrm{eV}$; however, in the case of the $\mathrm{Cu} L M M$ region where the position of this peak is of analytical importance for the assignment of oxidation state, the step size is $0.1 \mathrm{eV}$. 


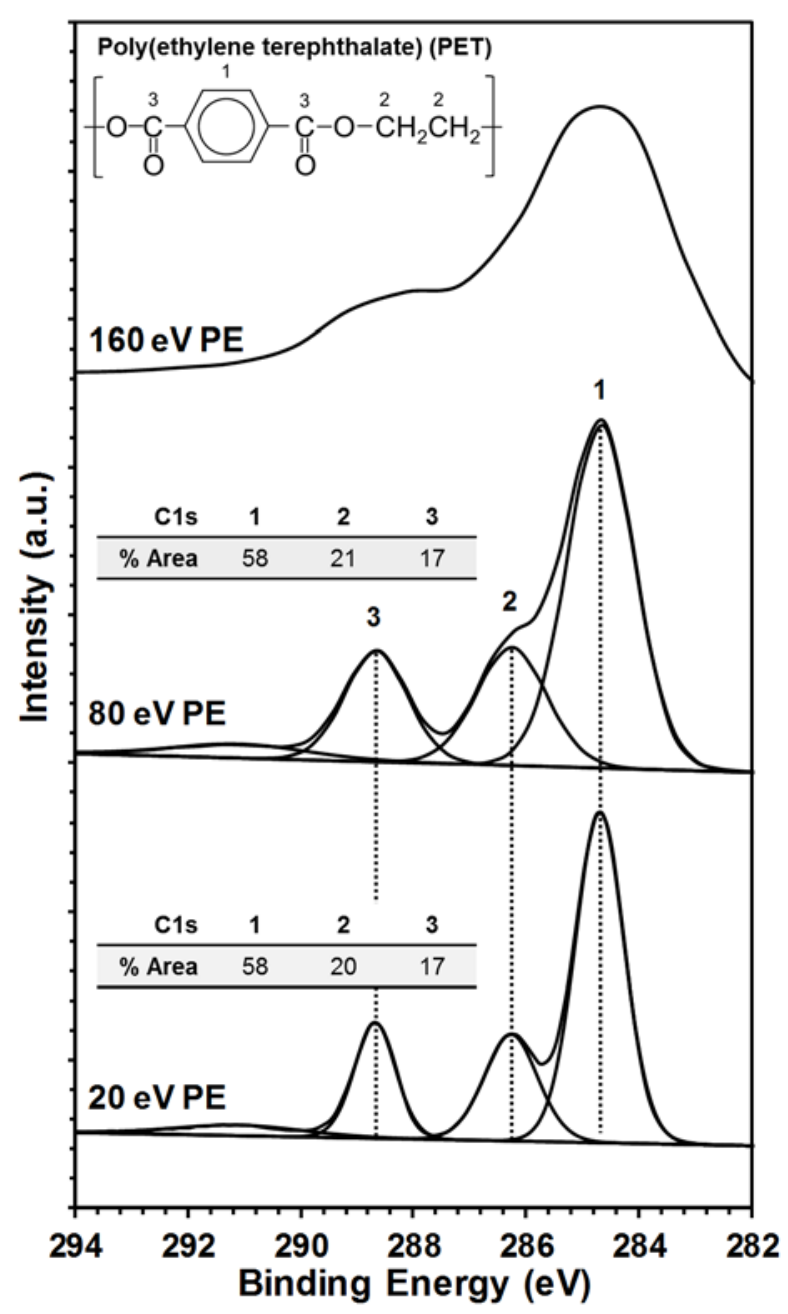

Figure 3 - Peak fitting regions from the $80 \mathrm{eV}$ pass energy St Malo survey spectrum for poly(ethylene terephthalate) shows excellent agreement with the high-resolution $\mathrm{C} 1 \mathrm{~s}$ region collected at $20 \mathrm{eV}$ pass energy.

Survey spectra are collected from $1350 \mathrm{eV}$ (binding energy) to $-6 \mathrm{eV}$ for conducting surfaces or $-10 \mathrm{eV}$ for insulators (to allow for 'over compensation' from the neutralisation system). High-resolution spectra are collected such that an entire region is covered, including both spin-orbit split components for $\mathrm{p}, \mathrm{d}$ and f orbitals. A further $30 \mathrm{eV}$ scan range is added (or more as necessary) from the highest binding energy component to encompass the background and any loss structure after the peak. The valence band is typically collected from 40 to $-10 \mathrm{eV}$, although may be modified in range depending on presence of other features. 
Survey spectra are collected in a single scan, while other photoelectron regions are collected until a sufficient signal to noise ratio (SNR) is achieved.

The setting of the correct analysis height for each sample is important for optimal performance of the spectrometer. While both the AXIS Nova and Ultra have automated routines for finding the correct analysis height, a manual procedure is adopted for the database to ensure consistency. The sample surface is first positioned at the optical focal point of the instrument microscope, and an optimal lateral position is found (away from obvious defects and sample edges). Snapshot spectra collected at $600 \mathrm{eV}$ (PE $160 \mathrm{eV}$ giving a $13.8 \mathrm{eV}$ window) are integrated for total counts over a $1000 \mathrm{~ms}$ dwell time. The $600 \mathrm{eV}$ binding energy region is chosen because it tends to provide a featureless background region of the spectrum. The X-ray source is operated at $75 \mathrm{~W}(15 \mathrm{kV}, 5 \mathrm{~mA})$ and for insulators the neutraliser is active. The sample $\mathrm{z}$ position is moved in $50 \mu \mathrm{m}$ steps above and below the optical focal point while the total integrated counts is monitored. The final $\mathrm{z}$ position is the median value of those that provide the greatest count rates.

The collection of spectra from metal foils necessitates the use of in situ ion beam cleaning to remove surface contamination and oxides. Both XPS instruments employed in this work utilise monoatomic argon ion sources for in situ cleaning, the primary difference between the two being that the Minibeam IV (on the AXIS Nova) has a floating mode for low-energy (down to $100 \mathrm{eV}$ ) sputtering. For oxide removal, a large raster size of $3 \times 3 \mathrm{~mm}^{2}$ is chosen to ensure that the analytical area of $300 \times 700 \mu \mathrm{m}^{2}$ does not impinge upon the edges of the ion-etched crater. The maximum ion energy of $4 \mathrm{keV}$ is chosen, with a beam current of $1.5 \mu \mathrm{A}$ at the sample surface. Etching is continued until the oxide layer signal is sufficiently removed from the spectrum.

The AXIS instruments utilise a low-energy electron source for charge neutralisation of insulating and poorly conducting surfaces. Three parameters are available to the analyst for optimising charge neutralisation performance: filament current, charge balance, and filament bias. Default parameters on our instrument are $1.65 \mathrm{~A}, 3.5 \mathrm{~V}$ and $1.1 \mathrm{~V}$ respectively. In most cases, adjustment of the charge balance voltage is all that is necessary to achieve good neutralisation, with typical values ranging from $2.8-4.2 \mathrm{~V}$. To obtain the optimal settings for 
a given surface, C 1s spectra are collected at PE $20 \mathrm{eV}$ from $295-275 \mathrm{eV}$ with relatively short dwell time ( $\leq 100 \mathrm{~ms})$. The AXIS neutraliser often 'over-compensates' surfaces, that is, shifts peaks to lower binding-energy values than would be expected, so absolute peak position is not used to identify optimal neutralisation. Instead, the best settings are those for which the expected peak shape is optimised. For adventitious carbon contamination, optimum peak shape is when the FWHM of the C1s line-shape is at its lowest value (the sharpest peak shape) with no asymmetry in the main component on either the low or high binding-energy sides, and no other obvious artefacts. For polymer samples, a priori knowledge of the expected C1s peak shape is ideal, as this provides a reference for 'good' neutralisation. The protocol remains the same, however. The narrowest peak shape is chosen as indicative of the best settings, but with additional focus on the ability to resolve different chemical states in the $\mathrm{C} 1 \mathrm{~s}$ line-shape. In the absence of a $\mathrm{C} 1 \mathrm{~s}$ signal other peaks can be chosen, preferably those that are expected to be narrow and symmetric. The binding energy scales for datasets in XPSSurfA are not corrected for charge shifts before being uploaded to the database. The expectation is that users would perform any corrections using the same method as applied to their own data, and that these corrections are detailed fully in publications so that they can be replicated by other users.

\subsection{Metadata Description}

Database entries in XPSSurfA are described by a rich metadata schema, presented in Table 4. Elements in the metadata are captured to describe in detail the origin of the materials, how they were prepared for analysis, how the data were acquired, the analyst involved in the work, and how and where the data are stored. Like the sample preparation and data acquisition protocols, the metadata description was formulated early in the development of XPSSurfA to act as a blueprint for all future work on the database structure and the collection of the data. As a whole, this metadata description satisfies completely section 4.2 (Analyst's Record) of ISO 16243:2011.

The metadata schema is informed by the ISO 14976:1998 and 16243:2011 standards, with additional elements related specifically to this database. Table 4 relates each metadata 
element in XPSSurfA to the relevant elements described in these standards, the source of this information within our system, and a comment about the metadata element.

Foremost, an integer identifier is assigned to each record automatically when the record is uploaded to XPSSurfA. The number is unique, is never reused in the system, and this metadata element persists in the database even if a record is deleted. Additionally, entries will have a digital object identifier (DOI) automatically generated to enable records to be found and cited through online search engines in perpetuity.

Elements exist in the description to enhance findability of the database. These include a reference to the journal citation that describes the database in full (this article), acknowledgement text that details the funding bodies involved in the creation of the database, and the license under which the data are shared. Also included are links to the protocols that governed the sample handling and data acquisition, stored on the ANFFhub sister-site, as a means of interconnecting the ecosystems of CMSShub and ANFFhub. Each record then has a simple summary element to tell the reader what data are contained within the record, and a reference to the original creator of the record and the data. The data file name and its format are also considered important metadata elements and are included in the description.

A rich metadata description requires that materials entered into the database have a clear and well-described provenance. For purchased reference materials, this includes the name of the supplier, the supplier catalogue number for the material, batch number for the specimen under analysis (whenever possible), the Chemical Abstracts Service identifier (CAS ID) and physical information such as purity and dimensions. When a record is created in XPSSurfA this information is sourced from the RLI surface analysis record. Information about the material to be analysed is entered by the analyst into the 'General Description' field of the RLI surface analysis request (which carries through to the record, see supplementary information Figures S1 and S2) using specific syntax to enable the metadata to be easily parsed by the database and included in the metadata fields.

The acquisition of the data is described completely, with information collected from both the RLI and the converted VAMAS file. Only two XPS instruments were used in the creation of 
this database, for which much of the experimental information is reported within the VMS file. Details are also included in the RLI for internal auditing purposes, and so a level of redundancy exists for some metadata elements. Some elements, such as the electron take-off angle, the analyser acceptance angle and the analysis source angle are not carried across to the VMS file upon conversion. These are constant for most records, and are thus entered automatically and only changed should it be necessary.

Elements are also added to enable interconnectivity between XPSSurfA and other resources. These include a simple 'Related links' field that could act as a connection between related records in a database (for example, records for copper metal and its oxides $\mathrm{Cu}_{2} \mathrm{O}$ and $\mathrm{CuO}$ could be linked together), or could act as a portal out to other databases or websites relevant to the record, the database, or surface analysis in general. The ability to include links to publications making use of particular records in XPSSurfA, for example, would promote further usage of the data, and act as an extra avenue for exposure to a body of research. A metadata field specific to the Australian research environment, 'Associated subject types', allows records to be tagged using Fields of Research (FOR) codes that are listed under the Australian and New Zealand Standard Research Classification (ANZSRC), further enhancing the connectivity of the database.

\subsection{Data transfer formats}

With a suite of instrumentation and the very high throughput that modern XPS instruments afford, a great volume of data can be generated at CMSS on a daily basis. These data can range from simple wide-energy survey spectra at specified points on a surface, to complex, multi-dimensional sputter depth profiles with angle-resolved analysis. With each surface analysis vendor supplying their instruments with proprietary software for acquisition, the question that is inevitably asked is how does one easily store, compile and forward data to collaborators of CMSS, and users of the XPSSurfA database, in a standardised format and through a medium that is easily accessible and traceable?

Part of this problem can be solved by using a standard data format such as the widely accepted Surface Chemical Analysis Standard Data Transfer Format, described by 
ISO 14976:1998 ${ }^{15}$. This format was developed by a number of surface analysts and manufacturers within the Versailles Project on Advanced Materials and Standards (VAMAS) community ${ }^{24}$. The binary data formats generated from instruments can be converted into this standard format through intermediary applications often bundled with the instrument software. In the case of the AXIS instruments at CMSS, VAMAS files can be output directly from the Vision software package. VAMAS files generated from XPS analysis can then be viewed and processed within readily available software such as CasaXPS (Casa Software Ltd, Teignmouth, UK). Many Australian and international institutions involved in surface analysis hold site licenses for CasaXPS. Each file contains all the data necessary to have a firm grasp on how the spectra were collected including experimental parameters such as source energy and power, analyser pass energy, step size and dwell time. Further details such as tilt angles and etch times can also be included. This coupled with the metadata description that underpins all records in XPSSurfA, means that all details about a particular piece of analysis are transparent to the analyst.

In the computer science community there is a move away from text-based formats such as ISO 14976. This transition has not yet occurred in the surface analysis community. ISO 14976 will no doubt be superseded at some time, perhaps by a successor based on XML as proposed by Yoshikawa et al. ${ }^{25}$.

There remains value in the original binary file generated by a given surface analysis instrument. Frequently these files contain far more information than that present in converted text formats. The disadvantage is in the shareability of these files. Only those with the required software can read and interact with the files, which generally requires that the user also have the same (or similar) instrumentation from a given vendor or their data processing software. Nevertheless, sharing the original binary files alongside more widely accessible text formats allows users to compare reference data directly on their own instrumentation during an acquisition.

XPSSurfA makes available three data transfer formats: 
- the proprietary .dset binary file containing data as acquired from within the Kratos Vision software package,

- a text-based .vms (VAMAS) file exported from the .dset file within the Kratos Vision Processing software package,

- and a generated .xml file that is essentially the .vms file with each line in the file tagged with the ISO 14976 descriptor using XML syntax.

In this way, XPSSurfA is able to maximise the usability of its data. For researchers with access to similar instrumentation, the .dset file can be used directly on the instrument to enable immediate comparisons. The VAMAS file allows all other researchers to view the data within the powerful CasaXPS processing environment, and since the dominant surface analytical vendors all have a means to export or convert their data into a VAMAS compatible format, comparisons with acquired data are easy. Note that files are not converted to the intermediary .kal format before conversion to .vms. Finally, the inclusion of an XML based format gives XPSSurfA a certain level of 'future-proofing', an example of which is given in supplementary Figure S3.

\subsection{XPSSurfA as a FAIR Database}

XPSSurfA satisfies the requirements of a FAIR data framework ${ }^{14}$, one of the key goals in its development. Data on XPSSurfA are

- Findable - data are assigned unique persistent identifiers, described by rich metadata and are indexed in a searchable resource,

- Accessible - data are retrieved via their unique identifiers and their metadata using well-established protocols,

- Interoperable - data are stored using formalised standards that are broadly utilised in the surface analysis context and include references to related data and metadata,

- Reusable - metadata is rich, accurate and relevant to the database, with clear provenance and all data are published under a license that promotes usage. 
4. Using the Database

\subsection{Creative Commons License}

The XPS database is licensed under the Creative Commons (Version 4.0) open access framework ${ }^{26}$. Creative Commons provides a legal foundation for sharing material widely and under flexible and attractive terms. Version 4.0 licences are formulated in generic terms to be broadly acceptable in multiple international jurisdictions ${ }^{27}$. Creative Commons licensing is used widely for the sharing of internet content with notable examples including Wikipedia ${ }^{28}$, Flickr $^{29}$, YouTube ${ }^{30}$, and the Internet Archive ${ }^{31}$.

Formally, the licence type used for XPSSurfA is a Creative Commons, Attribution, NonCommercial Licence (CC BY-NC) (International Version 4.0). This licence requires those using the data to attribute its source and to acknowledge the funding agency inputs in the creation of the resource. All use under this licence must be non-commercial in nature.

This licence type was chosen to allow individual researchers free and flexible access to XPSSurfA, including ingesting the original data files into a data processing package of their choice, the opportunity to create a derivative version of the data file, for example by incorporating reference data into a publication figure, and publishing this information. An example of this latter point already exists in the literature, with XPSSurfA reference data being used to validate the presence of certain copper oxides in a deposited film ${ }^{32}$. We have limited use to non-commercial purposes to avoid free incorporation of the database into commercial products and to preserve the opportunity for CMSS to engage in commercial relationships in this area.

\subsection{Accessing XPSSurfA}

The landing page for XPSSurfA (msshub.latrobe.edu.au/xpsdatabase) is publicly accessible, while access into the database requires that a user create an account on the host site CMSShub (see Figure S4 in supplementary information). The impetus for this is to allow us to collect meaningful usage statistics for the database, and to alleviate the problem of automated scripts, bots and illegitimate users from harvesting information from the database. Account creation requires no more information than one would typically expect from such a 
system, with required fields including a name, password, contact email address (nominally with an institution domain) and an affiliation. Researchers within Australian academic institutions and government research agencies can easily use their institution credentials to login to and create an account on CMSShub, through the Australian Access Federation (AAF) 33. A notable addition is the inclusion of a valid ORCID (Open Researcher and Contributor ID) in the account creation. The ORCID is a unique identifier to find authors and publications online, and the deep and interactive nature of the HUBzero environment allows us to leverage this identifier and better connect XPSSurfA with research outputs that it has helped to create.

\subsection{Searching XPSSurfA and Displaying Spectra}

Once a user is logged into CMSShub and accesses XPSSurfA, they are presented with a list of all records currently in the database. The list is initially sorted alphabetically, but can be sorted in order of record ID, instrument name, CAS ID and the date created. From this list the user can simply browse the records and select one of interest, or alternatively narrow the selection by using the search functions on the right of the page. Here the database can be searched by the instrument used to create a record, X-ray source energy, or by entering search terms such as simple keywords (e.g. metal, powder, polymer) or the CAS ID specific to the material of interest. Figure 4 gives an example of this function, with the CAS ID for diamond entered as the search parameter and the resulting records matching this search term being displayed. Another option available to the user is to browse or search all spectra in the database by clicking 'Browse all Spectra'. An example is given in Figure S5 where the database is searched using ' $\mathrm{C} 1 \mathrm{~s}$ ' as the spectral search term, and thus only records with $\mathrm{C} 1 \mathrm{~s}$ spectra present are output and displayed.

Accessing the diamond record presents the user with available spectra for that record (Figure 5(a)), which can then be selected based on the spectra that are considered most important. The selected spectra are then viewed within the browser window (Figure 5(b)). The charting object is interactive, so the user can hover the pointer over data points and peaks to read off the binding energy and intensity values, or click to draw a box around a particular feature that upon release zooms in on that spectral feature. This is shown in Figure 5(b) and 
(c) for the C1s peak from diamond. Double-clicking on the chart then returns to the full view of the spectrum.

Links are available on these pages to view the licensing agreement (that is clearly displayed on all spectra using the relevant CC license badge), to access the ANFFhub experiment protocols, and to view the metadata for the sample and for each spectrum. A link is also present to download the data files for the entire record. Note that this is independent of the selected spectra, the data files that are downloaded are always the complete record.
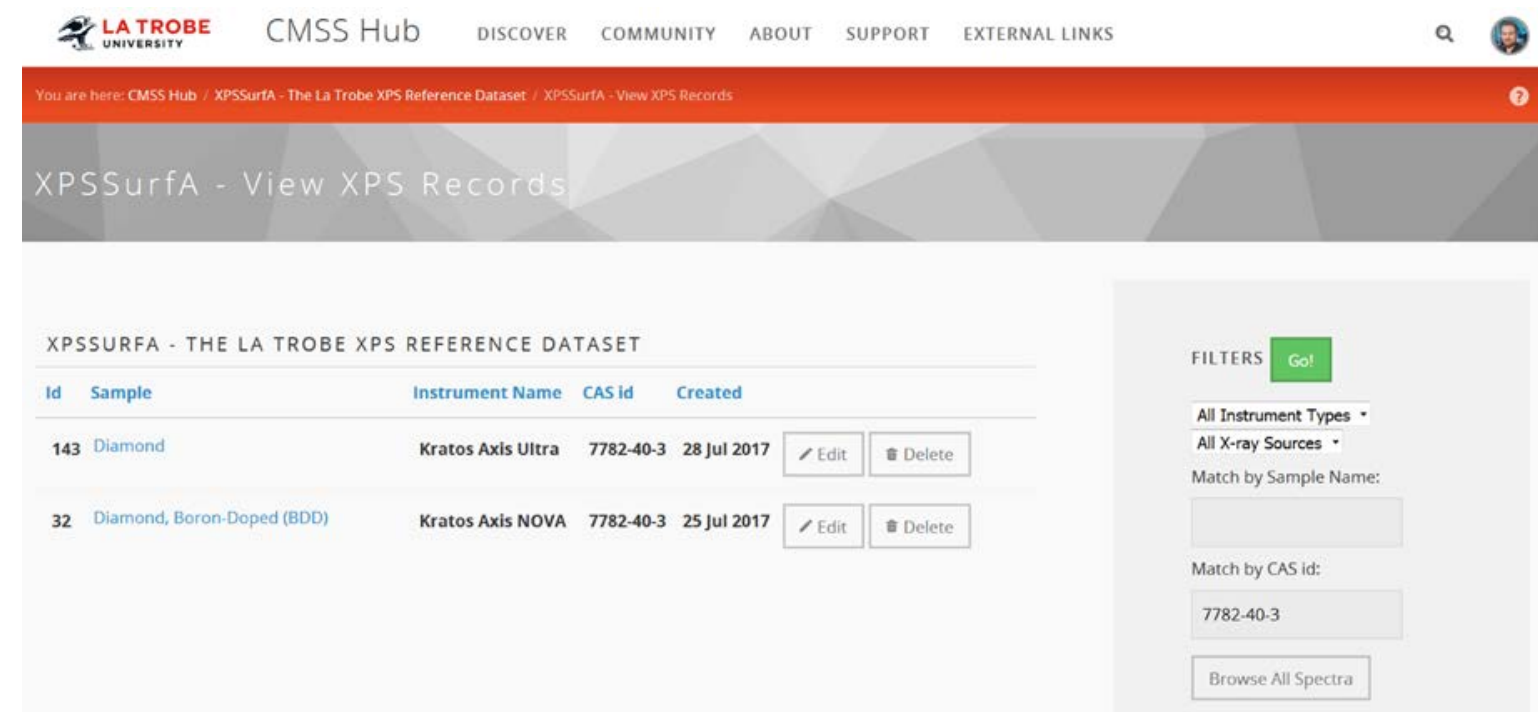

Figure 4 - The database can be searched based on instrument, X-ray source, or by keyword or CAS ID search. The CAS ID for diamond (7782-40-3) is entered as the search parameter and the resulting records that match the search criteria are displayed. 
a) Diamond

Summary: Diamond (CVD)

Preparation Ex-situ: Hydrogen plasma cleaned, mounted onto Ultra heating stub with tantalum mask, no adhesive

Preparation In-situ: Heated in situ to $350 \mathrm{C}$ for 4 hours

ANFFHub Experimental Protocols: 22232425 (must be logged in to anffhub.latrobe.edu.au)

\section{(C) (1) 8}

Download complete metadata for this dataset

Download VAMAS file in XML format and Raw Data

\section{AVAILABLE SPECIRA}

\begin{tabular}{|c|c|c|c|}
\hline Survey Spectra (eV per step) & Element/Transition & Element/Transition & Restrict to Pass Energy (eV) \\
\hline Whide (1) & ac is & $\Xi C K L L$ & 《 160 \\
\hline |v Wide (0.4) & & & 80 \\
\hline v Valence Band (0.2) & & & 20 \\
\hline View Spectra & & & 10 \\
\hline
\end{tabular}

b)

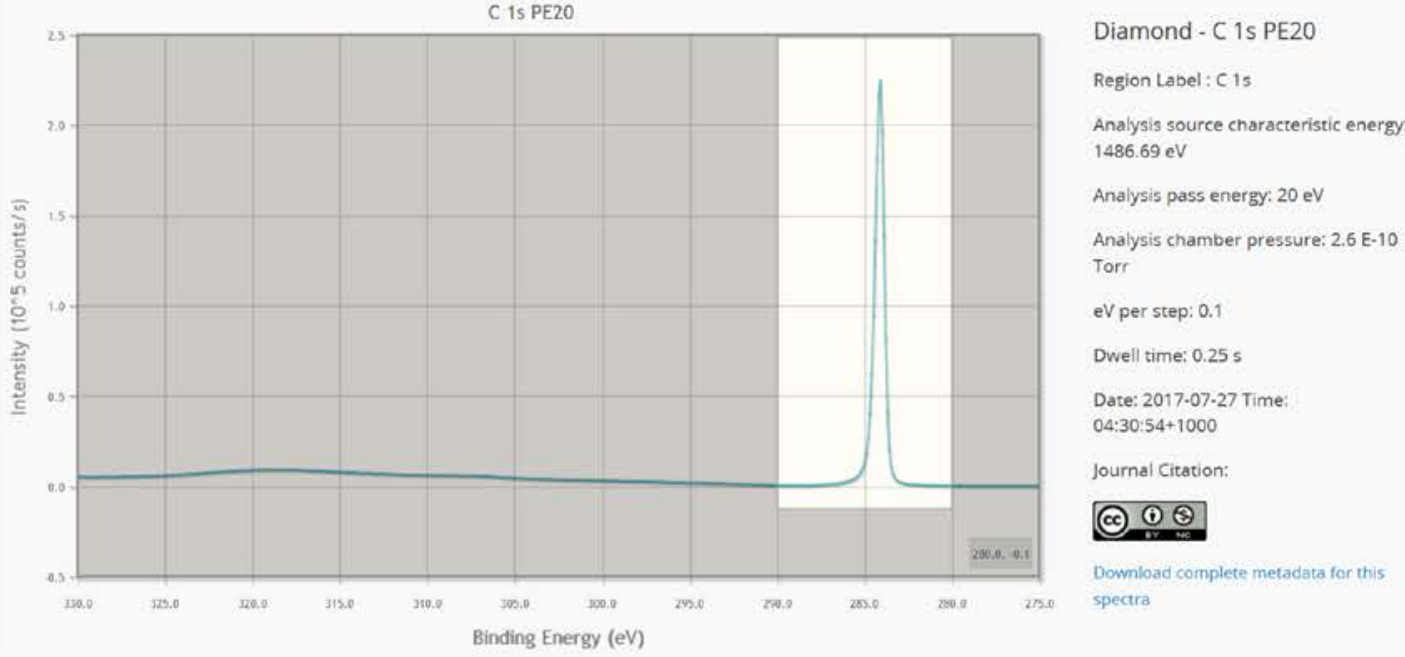

c)

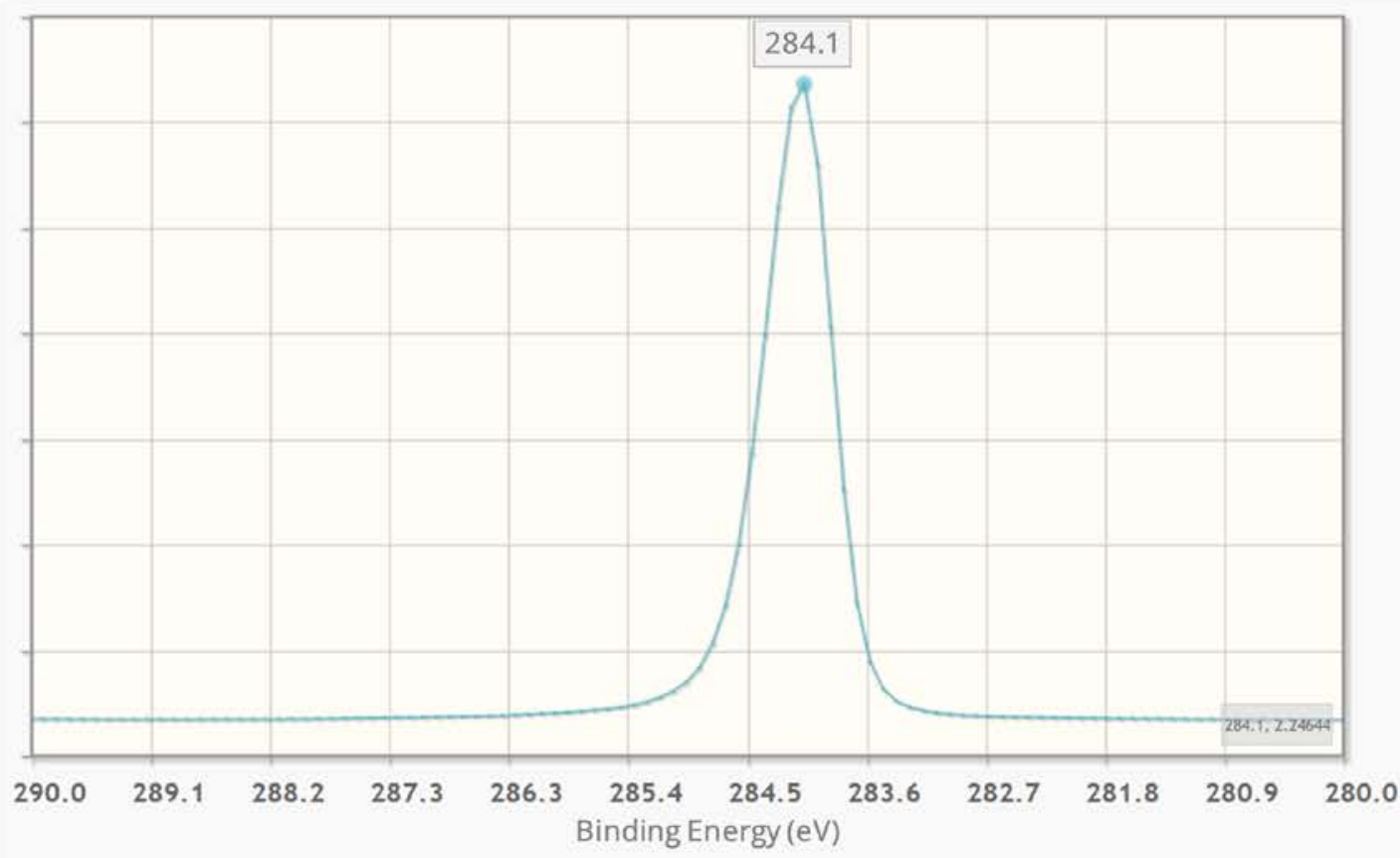

This article is protected by copyright. All rights reserved. 
Figure 5 - a) Selecting the Diamond record presents the user with the available spectra in the database, which can be chosen based on what is most useful to their research. b) The selected spectra are then displayed in the browser window and are interactive allowing for c) the user to zoom in on features and read off values precisely. 


\subsection{Downloading and Using Data}

Data files are available as a package in a zipped archive (.zip extension) containing the original .dset file, the converted .vms file, and the constructed .xml file. Since data on XPSSurfA are shared under Creative Commons licensing, these data can be used, remixed, and published by the user freely, provided it is not used for commercial purposes and that appropriate attribution is given to their source (i.e. XPSSurfA and its describing publication, this article). This means researchers can utilise the simple search and display functions of the database to find data that are relevant to their work, then download the data to their workspace to process and compare against and ultimately publish alongside their own data. An exemplar of this expected data usage already exists in the literature ${ }^{32}$.

\subsection{Analytics and Utilisation}

At the time of writing this article, XPSSurfA provides data from 113 materials comprising 1520 spectra. There are data from 16 pure metals, 20 compounds and oxides, 6 adhesive compounds and 69 polymers.

CMSShub and XPSSurfA are monitored using Google Analytics ${ }^{34}$, allowing CMSS to gauge the interaction and engagement that users are having with the database. Insightful information can be garnered from these analytics: from simple figures such as how many unique or returning visitors the site receives, to a more detailed view of the various modes of interaction with XPSSurfA including the number, type, complexity and duration of interactions during each visit. We can also gauge the impact of our interactions with the surface analysis community, with spikes in usage after publicity campaigns such as conference presentations, press releases and posts on social media.

XPSSurfA has been online for a period of 6 months. In that time, it has attracted 286 unique visitors from 34 countries. There have been more than 500 sessions logged, with $43 \%$ of those sessions from returning visitors. Many interactions are brief, with visitors spending less than a minute at the landing page and not creating an account to view the database. However, the visitors that do create an account and access XPSSurfA show significant engagement and 
retention, with most of these visitors spending between 3-30 minutes browsing the database and the majority of total page views being generated by these users (see Figure S6). 


\section{Conclusion and Outlook}

The XPSSurfA XPS database represents a rigorous, evolved and multi-faceted approach to collating, curating and sharing XPS data. It is structured to serve the needs of the surface analysis community, in particular, as large digital data repositories are established and data analysis moves into the "big data" realm. XPSSurfA is strongly standards-based and seeks to promote discussion in the community about the shape of future digital standards for the surface analysis community.

The data contained within XPSSurfA are closely described using carefully developed metadata description. The database adheres strongly to the concept of FAIR data: findable, accessible, interoperable and reusable.

XPSSurfA is readily scalable. Multiple growth directions are open, including expanding the core reference collection with additional data collected in house using CMSS instrumentation, incorporating additional reference data through a commissioning process or by collaboration using the protocols established in this study, exploring options for integrating existing databases under a common framework, or considering user-submitted data. The most robust approach will likely be the creation of several instances of the database to assist with curation and quality management.

The future of XPS data archives is likely to be cloud-based interoperable systems founded on common digital data standards. A fully harmonised approach to XPS data will ultimately allow universal sharing and utilisation, irrespective of originating instrument, data system, file format or laboratory. The fields of molecular biology and molecular spectroscopy offer examples of deep integration of great relevance for the surface analysis community. 


\section{Acknowledgements}

The Centre for Materials and Surface Science gratefully acknowledges the substantial contributions to the creation of the XPS Database by the staff of the La Trobe University Library, in particular, Andrew Williams, Clayton Bolitho, Rachel Salby, Eva Fisch and Simon Huggard, by the staff of the La Trobe University Information and Communications Technology (ICT) Division, in particular, Benji Wakely, and by the staff of the Australian National Data Service (ANDS), in particular, Kathryn Unsworth and Angeletta Leggio. This project was financially supported by a grant from the Australian National Data Service (Agreement HVC02). The authors acknowledge the significant contributions of Conal Tuohy, Daniel Tosello and Mark Kosten in a predecessor ANDS project conducted by La Trobe University. AJB thanks Peter Cumpson and Adam Roberts for many insights and enriching conversations that helped to develop this article and the thinking behind the database.

This research was supported by use of the NeCTAR Research Cloud and storage provided by Research Data Services (RDS), collaborative Australian research platforms supported by the National Collaborative Research Infrastructure Strategy. This work was performed in part at the Victorian node of the Australian National Fabrication Facility (ANFF), a company established under the National Collaborative Research Infrastructure Strategy to provide nano and micro-fabrication facilities for Australia's researchers, through the La Trobe University Centre for Materials and Surface Science. 


\section{References}

1. Linstrom PJ, Mallard WG. NIST Chemistry WebBook, NIST Standard Reference Database Number 69. http://webbook.nist.gov/. Accessed August 3, 2017.

2. Wagner CD. NIST X-ray Photoelectron Spectroscopy Database v1.0. 1989; http://srdata.nist.gov/xps/.

3. Naumkin AV, Kraut-Vass A, Gaarenstroom SW, Powell CJ. NIST X-ray Photoelectron Spectroscopy Database v4.1. 2012; http://srdata.nist.gov/xps/. Accessed August 3, 2017.

4. Benoit R. LaSurface. http://lasurface.com/accueil/index.php. Accessed August 3, 2017.

5. Mack P. (ed.), XPS Knowledge Base. http://xpssimplified.com/knowledgebase.php. Accessed August 18, 2017.

6. Moulder JF, Stickle WF, Sobol PE, Bomben KD. Handbook of X-ray Photoelectron Spectroscopy. 2nd ed: Perkin-Elmer Corporation, MN; 1992.

7. Beamson G, Briggs D. High resolution XPS of organic polymers: the Scienta ESCA300 database. John Wiley \& Sons Ltd; 1992.

8. XPS of Polymers Database. http://surfacespectra.com/xps/. Accessed January 15, 2017.

9. Crist BV. XPS International. http://xpsdata.com/. Accessed November 7, 2017.

10. eSpectra: Surface Science. http://espectrasurfacescience.com/. Accessed November 7, 2017.

11. Common Data Processing System (COMPRO). http://www.sasj.jp/COMPRO/. Accessed November 7, 2017.

12. CMSShub. http://cmsshub.latrobe.edu.au. Accessed November 19, 2017.

13. HUBzero. https://hubzero.org/. Accessed November 19, 2017.

14. Wilkinson MD, Dumontier M, Aalbersberg IJ, et al. The FAIR Guiding Principles for scientific data management and stewardship. Scientific Data. 2016;3:160018.

15. ISO 14976:1998 - Surface chemical analysis - Data transfer format. 1998; Retrieved from https://www.iso.org/standard/24269.html. Accessed January 15, 2017.

16. National eResearch Collaboration Tools and Resources project (Nectar). https://nectar.org.au/. Accessed December 18, 2017.

17. Touhy C. VAMASXML. https://github.com/conal-tuohy/ands-latrobe/wiki/VAMASXML. Accessed December 18, 2017.

18. ISO 15472:2001 - Surface chemical analysis - X-ray photoelectron spectrometers Calibration of energy scales. 2001; Retrieved from https://www.iso.org/standard/55796.html. Accessed January 15, 2017.

19. ISO 18116:2005 - Surface chemical analysis - Guidelines for preparation and mounting of specimens for analysis. 2005; Retrieved from https://www.iso.org/standard/29613.html. Accessed January 15, 2017.

20. ISO 16243:2011 - Surface chemical analysis - Recording and reporting data in X-ray photoelectron spectroscopy (XPS). 2011; Retrieved from https://www.iso.org/standard/30222.html. Accessed January 15, 2017. 
21. ISO 18115:2001 - Surface chemical analysis - Vocabulary. 2001; Retrieved from https://www.iso.org/standard/29189.html. Accessed January 15, 2017.

22. Castle JE. Developments in expert systems for automatic examination of samples by $\mathrm{X}$-ray photoelectron spectroscopy. Journal of Electron Spectroscopy and Related Phenomena. 2010;178-179(Supplement C):347-356.

23. Castle JE, Powell CJ. Report on the 34th IUVSTA workshop 'XPS: From Spectra to Results-Towards an Expert System'. Surface and Interface Analysis. 2004;36(3):225-237.

24. Dench WA, Hazell LB, Seah MP. VAMAS Surface Chemical-Analysis Standard Data Transfer Format with Skeleton Decoding Programs. Surface and Interface Analysis. 1988;13(2-3):63-122.

25. Yoshikawa H, Yoshihara K, Watanabe D, Tanaka H, Tanuma S. Proposal for common data transfer format for simulation softwares used in surface electron spectroscopies. Surface and Interface Analysis. 2014;46(10-11):931-935.

26. Creative Commons. https://creativecommons.org/licenses/by-nc/4.0/. Accessed December 14, 2017.

27. Peters D. CC's Next Generation Licenses - Welcome Version 4.0! https://creativecommons.org/2013/11/25/ccs-next-generation-licenses-welcomeversion-4-0/. Accessed December 14, 2017.

28. Wikipedia. https://www.wikipedia.org/. Accessed December 14, 2017.

29. Flickr. https://www.flickr.com/. Accessed December 14, 2017.

30. YouTube. https://www.youtube.com/. Accessed December 14, 2017.

31. Internet Archive. https://archive.org/. Accessed December 14, 2017.

32. Alajlani Y, Placido F, Barlow A, et al. Characterisation of $\mathrm{Cu}_{2} \mathrm{O}, \mathrm{Cu}_{4} \mathrm{O}_{3}$, and $\mathrm{CuO}$ mixed phase thin films produced by microwave-activated reactive sputtering. Vacuum. 2017; 144:217-228.

33. Australian Access Federation (AAF). https://aaf.edu.au/. Accessed December 14, 2017.

34. Features - Google Analytics. http://www.google.com/analytics/features/. Accessed December 15, 2017. 
Table 1 - Energy scale calibration results of both XPS spectrometers at CMSS, values given in $\mathrm{eV}$ (binding energy).

\begin{tabular}{|c|c|c|c|}
\hline Instrument & $\operatorname{Au} \mathbf{4 f}_{7 / 2}$ & $\operatorname{Ag~} 3 d_{5 / 2}$ & $\mathrm{Cu} 2 \mathbf{p}_{3 / 2}$ \\
\hline AXIS Nova & 84.06 & 368.21 & 932.70 \\
\hline AXIS Ultra & 84.01 & 368.21 & 932.67 \\
\hline
\end{tabular}

This article is protected by copyright. All rights reserved. 
Table 2 - Energy resolution calibration results of both XPS spectrometers at CMSS, values given in $\mathrm{eV}$, measured as FWHM of $\mathrm{Ag} 3 \mathrm{~d}_{5 / 2}$ peak at an analyser pass energy of $5 \mathrm{eV}$.

\begin{tabular}{cc} 
Instrument & FWHM Ag $\mathbf{3 d}_{\mathbf{5 / 2}}$ \\
\hline AXIS Nova & 0.50 \\
AXIS Ultra & 0.48 \\
\hline
\end{tabular}

This article is protected by copyright. All rights reserved. 
Table 3 - Data acquisition protocol for the AXIS Nova and Ultra instruments

\section{Parameter}

\begin{tabular}{|c|c|c|}
\hline \multirow{2}{*}{\multicolumn{2}{|c|}{$\begin{array}{l}\text { System base pressure } \\
\text { Acquisition pressure }\end{array}$}} & $<5 \times 10^{-10} \mathrm{mbar}$ \\
\hline & & $<1 \times 10^{-8} \mathrm{mbar}$ \\
\hline \multicolumn{2}{|l|}{ X-ray source } & Monochromatic Al K $\alpha$ \\
\hline \multicolumn{2}{|l|}{ X-ray energy } & $1486.69 \mathrm{eV}$ \\
\hline \multicolumn{2}{|l|}{ X-ray source voltage } & $15 \mathrm{kV}$ \\
\hline \multicolumn{2}{|l|}{ X-ray source emission } & $15 \mathrm{~mA}$ (metals), $10 \mathrm{~mA}$ (organics) \\
\hline \multicolumn{2}{|l|}{ X-ray source power } & $225 \mathrm{~W}$ (metals), $150 \mathrm{~W}$ (organics) \\
\hline \multicolumn{2}{|l|}{ Analyser mode } & FAT \\
\hline \multicolumn{2}{|l|}{ Lens mode } & FoV1 for AXIS Nova, Hybrid/FoV1 for AXIS Ultra \\
\hline \multicolumn{2}{|l|}{ Iris/aperture } & Slot (analytical area $300 \mu \mathrm{m} \times 700 \mu \mathrm{m})$ \\
\hline \multirow[t]{4}{*}{ Pass energy } & Standard survey & $160 \mathrm{eV}$ \\
\hline & St Malo survey & $80 \mathrm{eV}$ \\
\hline & Regions & $40 / 20 / 10 /(5) \mathrm{eV}$ \\
\hline & Auger regions, valence band & $20 \mathrm{eV}$ \\
\hline \multirow[t]{3}{*}{ Energy sweep } & Surveys & -6 to $1350 \mathrm{eV}$ ( -10 to $1350 \mathrm{eV}$ for insulators) \\
\hline & Regions & Encompass entire region $+30 \mathrm{eV}$ tail from highest $\mathrm{BE}$ component \\
\hline & Valence band & Typically -10 to $40 \mathrm{eV}$ \\
\hline \multirow[t]{5}{*}{ Step size } & Standard survey & $1.0 \mathrm{eV}$ \\
\hline & St Malo survey & $0.4 \mathrm{eV}$ \\
\hline & Regions & $0.1 \mathrm{eV}$ \\
\hline & Auger regions & $0.2 \mathrm{eV}$ or $0.1 \mathrm{eV}$ for analytical peaks (e.g. $\mathrm{Cu} L M M$ ) \\
\hline & Valence band & $0.2 \mathrm{eV}$ \\
\hline Dwell time & Surveys & $250 \mathrm{~ms}$ \\
\hline
\end{tabular}

This article is protected by copyright. All rights reserved. 


\begin{tabular}{lll} 
& Regions & $250 \mathrm{~ms}$ \\
Sweeps & Surveys & 1 sweep \\
& Regions & As many as required for good SNR \\
& Auger regions & 30 sweeps \\
& Valence band & 50 sweeps \\
\hline
\end{tabular}

This article is protected by copyright. All rights reserved. 
Table 4 - Complete metadata description for the XPSSurfA database with reference to the relevant ISO standards

Metadata Elemen

Record ID

Digital object identifier

Usage license

Summary

Created by

Journal citation

Acknowledgement

ANFFhub protocols

Data file

Data file format

Complete description

Sample ID

CAS ID

Dimensions

Supplier

Supplier's product code

Sample catalogue number Purity

Preparation ex-situ

Preparation in-situ

Collector list

Date

Name of instrument

X-ray source used

Analysis source energy

Analysis source strength

Analyser pass energy

Source angle

Analysis chamber pressure

Start energy

End energy

eV per step

Dwell time

Analyser axis take-off angle
ISO 14976 Reference ISO 16243 Reference

experiment i.d

operator i.d.

4.2.1.e - Name of analyst, and the analyst's affiliation

format identifier

sample i.d.

sample i.d.

sample i.d.

operator i.d.

year/month/day

instrument model i.d.

source

source energy

source strength

analyser pass energy

analysis source angle

abscissa start

abscissa increment

signal collection time

analyser axis take off 4.2.1.c - Description of the specimen before (and after) analysis

4.2.1.b - Unique specimen number

4.2.1.b - Unique specimen number

4.2.1.c - Description of the specimen before (and after) analysis

4.2.1.a - Name of the originating laboratory supplying the specimen

4.2.1.b - Unique specimen number

4.2.1.b - Unique specimen number

4.2.1.c - Description of the specimen before (and after) analysis

4.2.1.f - Details concerning ex situ specimen preparation before analysis

4.2.1.g - Details concerning in situ specimen preparation before analysis

4.2.1.e - Name of analyst, and the analyst's department and affiliation

4.2.1.d - Date of measurement(s)

4.2.2.a - Name or identification of equipment used

4.2.2.b - X-ray source used

$4,2,2 \mathrm{~b}$ - X-ray source used

4.2.2.c - X-ray power

4.2.2.e - Analyser pass energy (in eV)

4.2.2.f - The geometry of irradiation

4.2.2.h - Analysis chamber pressure before and during analysis

4.2.2.j - Start energy

4.2.2.k - End energy or scan width

4.2.2.1 - Number of data points

4.2.2.m - Acquisition time

4.2.2.g - Take-off angle used for the measurement
Automatically generated

Automatically generated

Manual entry constant

RLI

RLI

Manual entry constant

Manual entry constan

Manual entry constant

Instrument

RLI

RLI

RLI

RLI

RLI

RLI

RLI

RLI

RLI

RLI

RLI and VMS file

RLI

RLI and VMS file

VMS file

RLI and VMS file

VMS file

Automatic entry constan

RLI

VMS file

Calculated

VMS file

VMS file

Automatic entry constant
Comment

Integer value assigned upon creation of record and never reused

Unique DOI for each record to enable findability

The license under which the data are shared (CC BY-NC 4.0 International)

Entered into the RLI by the analyst upon creation of the reques

Taken from the RLI logsheet for the given analysis request

Journal citation for the article describing the database in full

Acknowledgment text for contributing funding sources

Fixed protocols determined at start of database creation

Kratos .dset file, converted .vms file, generated .xml file

*.dset/*.vms/*.xml

Collected from the RLI logsheet

Entered into the RLI by the analyst upon creation of the reques

Entered into the RLI by the analyst upon creation of the reques

Entered into the RLI by the analyst upon creation of the request

Entered into the RLI by the analyst upon creation of the request

Entered into the RLI by the analyst upon creation of the request

Entered into the RLI by the analyst upon creation of the request

Entered into the RLI by the analyst upon creation of the request

Entered into the RLI by the analyst upon creation of the request

Entered into the RLI logsheet by the analyst upon collection of the data

Taken from the RLI logsheet for the given analysis request

Collected from the VMS file

Taken from the RLI logsheet for the given analysis request

Entered into the RLI logsheet by the analyst upon collection of the data

Collected from the VMS file

Entered into the RLI logsheet by the analyst upon collection of the data Collected from the VMS file

Fixed for all analyses in the database from a given instrument

Entered into the RLI logsheet by the analyst upon collection of the data

Value provided in the VAMAS file ("abscissa start" field) as kinetic energy

Calculated based on start energy, step size, and no. of data points in block

Collected from the VMS file

Collected from the VMS file as time per eV step

Specific to the analysis, manually edited if required

This article is protected by copyright. All rights reserved. 
Area of analysis

Charge compensation

Lens mode

analysis width $\mathrm{x} / \mathrm{y}$

analyser mode

Acceptance angle

Peak or region labels

block i.d.

Related links

Associated subject types 4.2.2.i - Area of analysis

4.2.2.n - Charge compensation conditions, if charge compensation is used 4.2.2.p - When an instrument can be operated in a variety of lens modes 4.2.2.o - The value of the acceptance angle for photoelectrons

4.3.a - Peak or region labels (e.g. C $1 \mathrm{~s}, \mathrm{Cu} 2 \mathrm{p} 3 / 2$ )

4.3.g - Any energy reference used

4.4.d - The sensitivity factors used

4.4.b - The name and version of the data processing software used
VMS file

RLI and VMS file

VMS file

Manual entry constant

VMS file

Manual entry constant

Manual entry constant

Manual entry constan

Manual entry

Manual entry constant
Collected from the VMS file comment lines

Specifies whether charge compensation is used and the relevant conditions Collected from the VMS file comment lines

Specific to the lens mode used, to be entered manually

Collected from the VMS file block I.D.

Placekeeper for future use

Placekeeper for future use

Fixed for all analyses in the database

To create links between database entries, protocols, or other objects Subject codes written into every database entry 

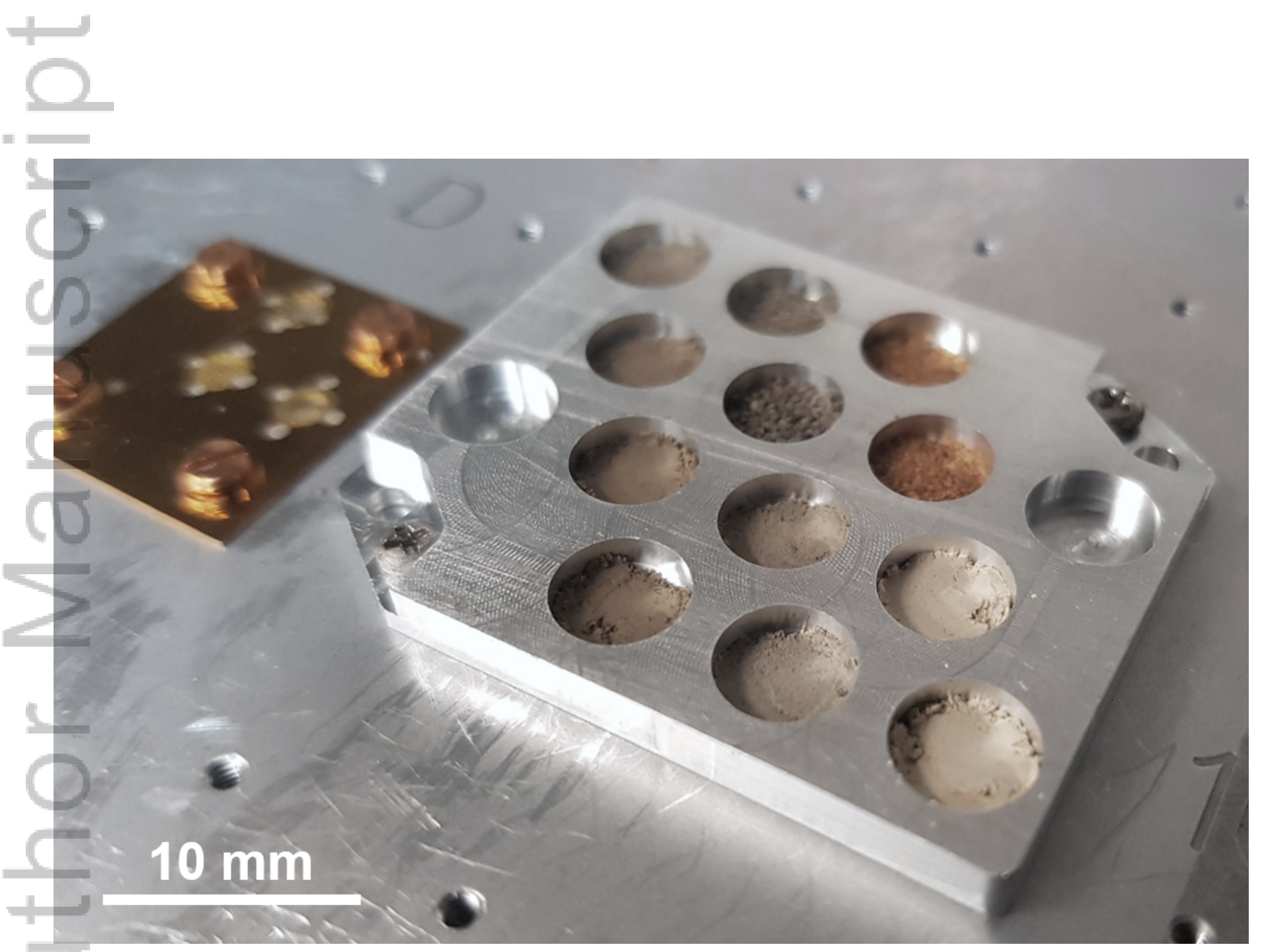

SIA_6417_F2.tif

This article is protected by copyright. All rights reserved. 


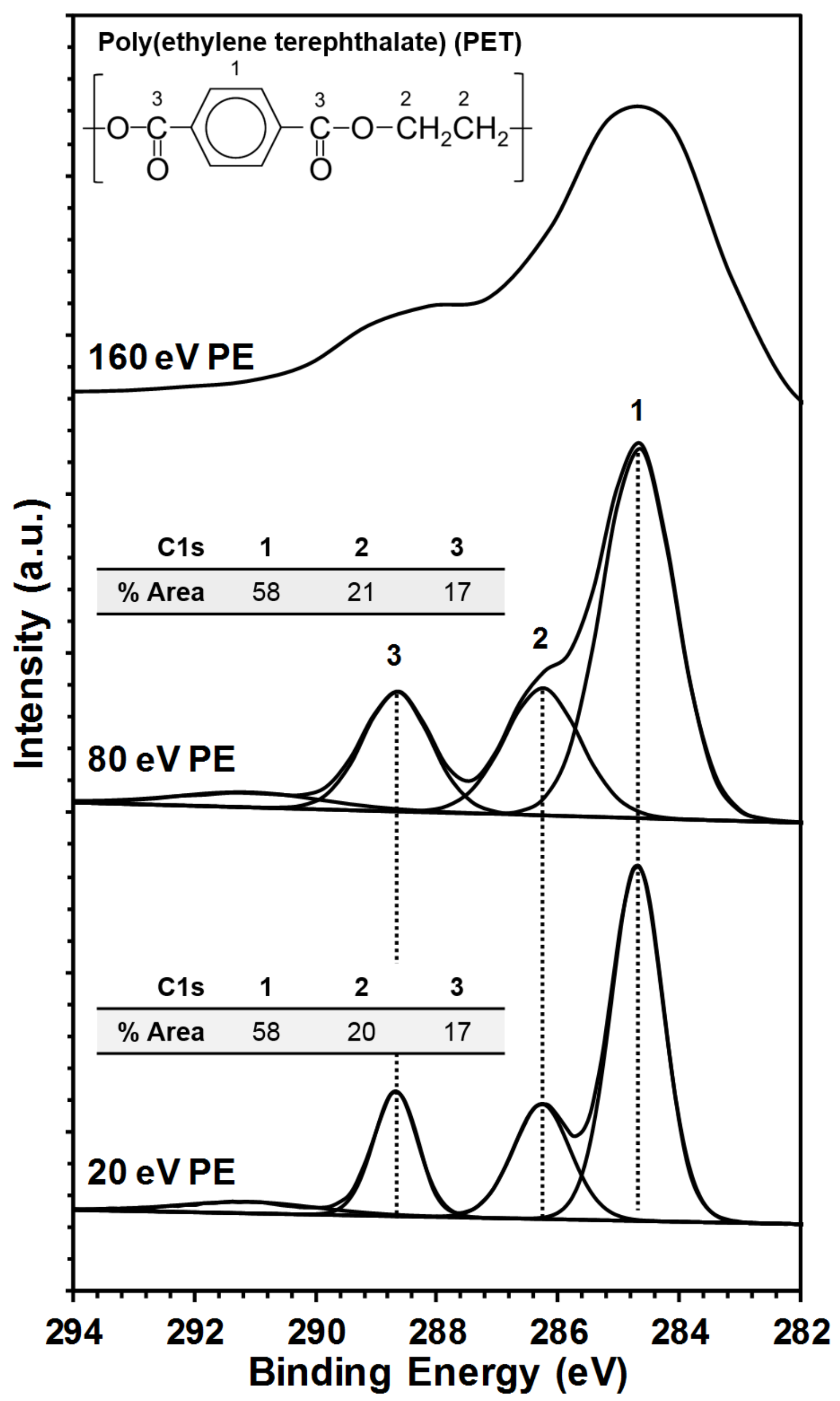

This article is protected by copyright. All rights reserved. 


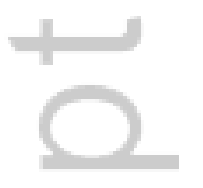

XPSSURFA - THE LA TROBE XPS REFERENCE DATASET

Id

143 Diamond

32 Diamond, Boron-Doped (BDD)
Instrument Name CAS i

Kratos Axis Ultra $\quad 7782-40-3 \quad 28$ Jul 2017

Kratos Axis NOVA 7782-40-3 25 Jul 2017
Edit

苗 Delete

Edit 畾 Delete

FILTERS Go!

All Instrument Types All X-ray Sources Match by Sample Name:

Match by CAS id: 7782-40-3

Browse All Spectra

SIA_6417_F4.tif

This article is protected by copyright. All rights reserved. 
a) Diamond

Summary: Diamond (CVD)

Preparation Ex-situ: Hydrogen plasma cleaned, mounted onto Ultra heating stub with tantalum mask, no adhesive Preparation In-situ: Heated in situ to $350 \mathrm{C}$ for 4 hours

ANFFHub Experimental Protocols: 22232425 (must be logged in to anffhub.latrobe.edu.au)

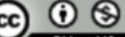

Download complete metadata for this dataset

Download VAMAS file in XML format and Raw Data

\section{AVAILABLE SPECTRA}

Survey Spectra (eV per step)

Element/Transition Element/Transition Restrict to Pass Energy (eV)

๑ Wide (1)

v C 1s

๑ C KLL

จ 160

\. Wide (0.4)

จ 80

๑ Valence Band (0.2)

จ 20

View Spectra

จ 10

จ 5

b)

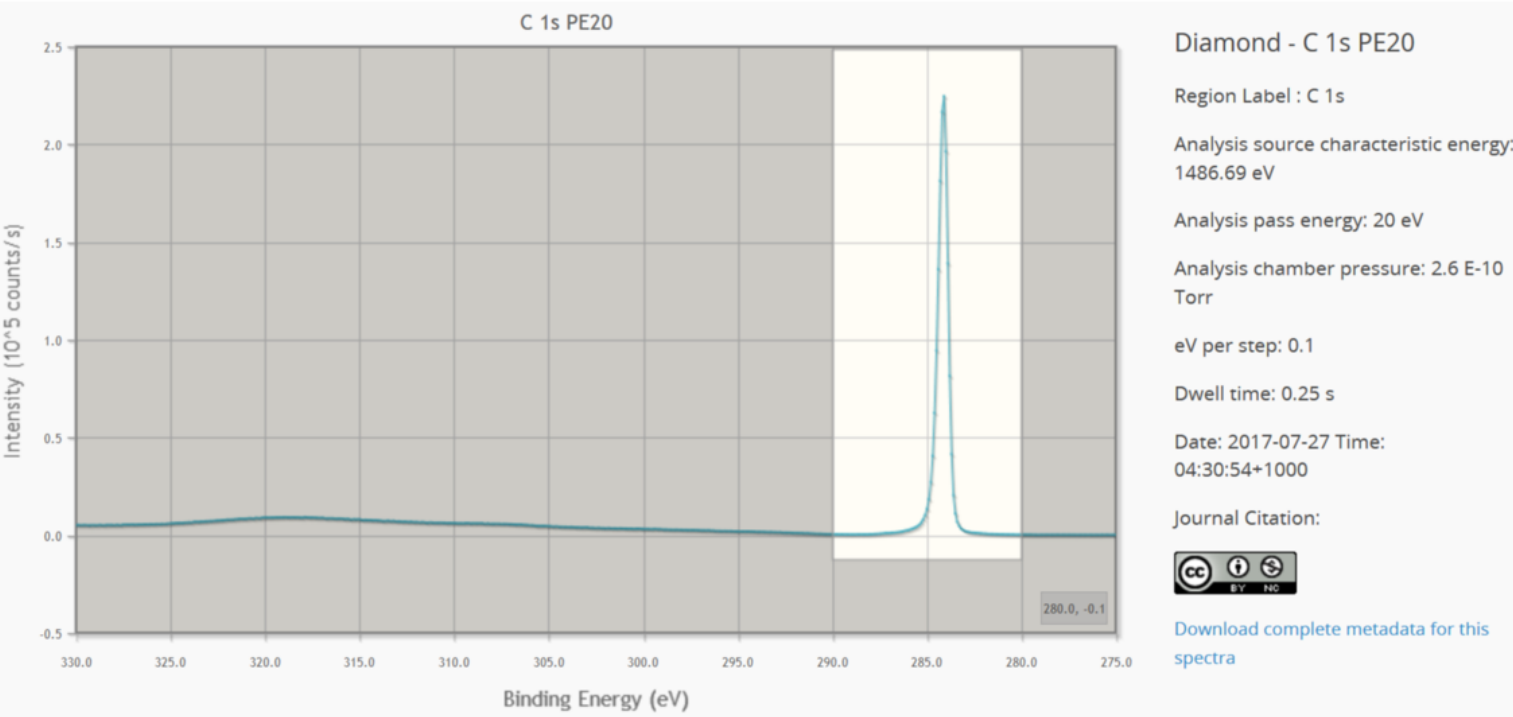

c)

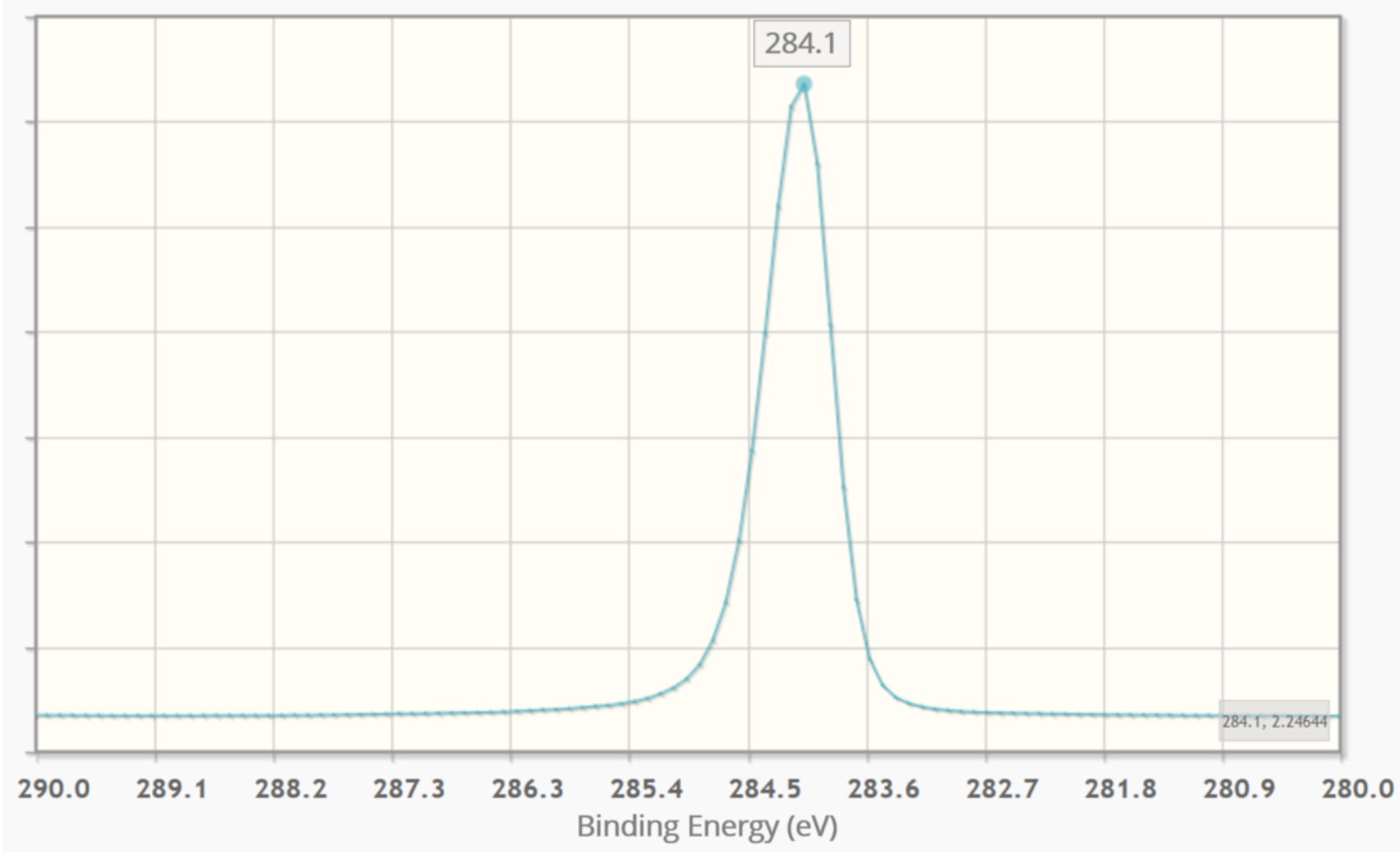

SIA_6417_F5.tif

This article is protected by copyright. All rights reserved. 


\section{University Library}

\section{- M M N E R VA A gateway to Melbourne's research publications}

Minerva Access is the Institutional Repository of The University of Melbourne

Author/s:

Barlow, AJ;Jones, RT;McDonald, AJ;Pigram, PJ

Title:

XPSSurfA: An open collaborative XPS data repository using the CMSShub platform

Date:

2018-05-01

Citation:

Barlow, A. J., Jones, R. T., McDonald, A. J. \& Pigram, P. J. (2018). XPSSurfA: An open

collaborative XPS data repository using the CMSShub platform. SURFACE AND INTERFACE ANALYSIS, 50 (5), pp.527-540. https://doi.org/10.1002/sia.6417.

Persistent Link:

http://hdl.handle.net/11343/284680 